\title{
Convergent Genomic Studies Identify Association of GRIK2 and NPAS2 with Chronic Fatigue Syndrome
}

\author{
Alicia K. Smith ${ }^{a, 1}$ Hong Fang ${ }^{b}$ Toni Whistler ${ }^{a}$ Elizabeth R. Unger ${ }^{a}$ \\ Mangalathu S. Rajeevan ${ }^{a}$ \\ ${ }^{a}$ Division of High-Consequence Pathogens and Pathology, Centers for Disease Control and Prevention, \\ Atlanta, Ga., and ' ${ }^{\mathrm{b} Z}$-Tech Corporation, an ICF International Company at NCTR/Food and Drug Administration, \\ Jefferson, Ark., USA
}

\section{Key Words \\ Chronic fatigue syndrome $\cdot$ Genome-wide association • \\ Gene expression - GRIK2 - NPAS2 - Glutamatergic \\ neurotransmission $\cdot$ Circadian rhythm $\cdot$ Orexin signaling}

\begin{abstract}
Background: There is no consistent evidence of specific gene(s) or molecular pathways that contribute to the pathogenesis, therapeutic intervention or diagnosis of chronic fatigue syndrome (CFS). While multiple studies support a role for genetic variation in CFS, genome-wide efforts to identify associated loci remain unexplored. We employed a novel convergent functional genomics approach that incorporates the findings from single-nucleotide polymorphism (SNP) and mRNA expression studies to identify associations between CFS and novel candidate genes for further investigation. Methods: We evaluated 116,204 SNPs in 40 CFS and 40 nonfatigued control subjects along with mRNA expres-
\end{abstract}

sion of 20,160 genes in a subset of these subjects (35 CFS subjects and 27 controls) derived from a population-based study. Results: Sixty-five SNPs were nominally associated with CFS ( $p<0.001)$, and 165 genes were differentially expressed ( $\geq 4$-fold; $p \leq 0.05$ ) in peripheral blood mononuclear cells of CFS subjects. Two genes, glutamate receptor, ionotropic, kinase 2 (GRIK2) and neuronal PAS domain protein 2 (NPAS2), were identified by both SNP and gene expression analyses. Subjects with the G allele of rs2247215 (GRIK2) were more likely to have CFS $(p=0.0005)$, and CFS subjects showed decreased GRIK2 expression ( 10 -fold; $p=0.015$ ). Subjects with the T allele of rs356653 (NPAS2) were more likely to have CFS $(p=0.0007)$, and NPAS2 expression was increased (10-fold; $p=0.027$ ) in those with CFS. Conclusion: Using an integrated genomic strategy, this study suggests a possible role for genes involved in glutamatergic neurotransmission and circadian rhythm in CFS and supports further study of novel candidate genes in independent populations of CFS subjects.
Copyright ๑ 2011 S. Karger AG, Basel

1 Current affiliation: Department of Psychiatry and Behavioral Sciences,
Emory University School of Medicine, Atlanta, Ga., USA.

\section{KARGER}

Fax +4161306 1234 E-Mail karger@karger.ch www.karger.com
(C) 2011 S. Karger AG, Basel

0302-282X/11/0644-0183\$38.00/0

Accessible online at: www.karger.com/nps
The findings and conclusions in this report are those of the authors and do not necessarily represent the views of the funding agency. 


\section{Introduction}

Recent years have witnessed a surge in interdisciplinary efforts to delineate the pathophysiology of chronic fatigue syndrome (CFS), a debilitating disorder of unknown etiology with no known lesions, biomarkers or laboratory tests for diagnosis [1]. Neuroendocrine and immune system deregulation as well as perturbations in cognitive, sleep and metabolic functions have been documented in CFS [1-3]. Further, twin, family and candidate gene studies support a moderate genetic contribution to the development of CFS [4-6]. Gene expression and candidate gene association studies support the association of several genes with CFS [4, 7-10], but candidate gene studies remain limited by the poor understanding of CFS pathophysiology. Despite progress, there is no consistent evidence of specific gene(s) or molecular pathways that contribute to CFS pathogenesis, provide insights for therapeutic intervention or contribute to diagnosis.

Polymorphisms are more likely to impact on gene expression than to change protein coding sequences, suggesting that gene expression may be an early manifestation of a complex phenotype [11]. Genetic associations supported by independent molecular evidence are more compelling than those identified by a single approach. Along these lines, analyses of genetic linkage or association results combined with mRNA expression have revealed promising candidates for schizophrenia [12, 13], obesity [14], cancer treatment outcome [15] and drug sensitivity [16]. Recently, the convergent functional genomics approach, which integrates multiple independent lines of evidence, was used successfully to prioritize viable candidate genes and blood biomarkers for multiple neuropsychiatric diseases, including bipolar disorder, psychosis and mood disorders [17-21].

To begin the process of identifying novel candidates for further study in CFS, we utilized a novel convergent functional genomics approach in a proof of principle exploration, by combining unbiased studies of polymorphisms and mRNA expression using subjects recruited from a population-based study. Since there are no known lesions in CFS, gene expression analysis was performed using total RNA from peripheral blood mononuclear cells (PBMCs), in keeping with the hypothesis that changes in PBMC expression reflect systemic changes due to illness [22]. We identified glutamate receptor, ionotropic, kinase 2 (GRIK2) and neuronal PAS domain protein 2 (NPAS2), genes involved in glutametergic neurotransmission and circadian rhythm regulation, respectively, to be consistently associated with CFS in independent ge- nomic analyses. These previously unrecognized associations should provide exciting new hypotheses in the study of CFS.

\section{Materials and Methods}

Subjects and Illness Classification

This study adhered to the human experimental guidelines of the US Department of Health and Human Services and the Helsinki Declaration. The Centers for Disease Control and Prevention (CDC) Human Subjects Committee approved the study protocol, and all subjects gave informed consent.

The subjects in this study were from the Wichita CFS Surveillance Study, a population-based study conducted between 1997 and 2000 [23]. From December 2002 to July 2003, 227 subjects were recruited from this surveillance cohort for a 2-day in-hospital study to reevaluate symptoms and exclusionary medical and psychiatric conditions and to collect samples for molecular epidemiologic studies. In brief, 43 subjects meeting the criteria for CFS and 60 control subjects were identified. CFS was diagnosed using the criteria of the 1994 International Research Case Definition [24] as recommended by the International CFS study group [25] and currently used by the CDC [23]. To identify exclusionary medical conditions, a standardized medical history and physical examination, review of current medications and routine blood and urine clinical laboratory tests were performed as recommended by the International CFS Study Group [23, 25]. Exclusionary psychiatric conditions were identified by specifically trained, licensed psychiatric interviewers using the Diagnostic Interview Schedule. The single-nucleotide polymorphism (SNP) association study was restricted to 40 CFS and 40 control Caucasian subjects because of limited power to detect associations in other racial groups. We also restricted the gene expression analysis to the subjects for whom RNA microarray data were available (35 CFS subjects and 27 controls).

\section{Isolation of DNA and RNA}

Peripheral blood was collected in 8-ml vacutainer tubes containing citric acid (BD, N.J., USA), and PBMCs were immediately isolated on Lymphocyte Separation Medium (Organon Teknika, N.C., USA). PBMCs were stored in liquid nitrogen under conditions designed to maintain viability. Both DNA and RNA were extracted from the same PBMC sample by a Trizol DNA/RNA extraction protocol (Invitrogen, Carlsbad, Calif., USA). Genomic DNA was amplified using the Genomiphi DNA amplification kit (Amersham Biosciences, Piscataway, N.J., USA). Amplified DNA was quantified by TaqMan PCR with primers and probe for $\beta$ globin ( 2 copies of $\beta$-globin/cell and 5 pg of DNA per cell) prior to genotyping. Contaminating DNA from RNA preparations was removed by digesting it with $2 \mathrm{U}$ of DNase I (GeneHunter Corporation, Tenn., USA) at $37^{\circ} \mathrm{C}$ for $15 \mathrm{~min}$. The quality and quantity of the RNA preparation was determined using a Bioanalyzer 2100 (Agilent, Calif., USA).

SNP Association Study

The Affymetrix GeneChip Mapping 100K Set (split into 2 array sets referred to as $50 \mathrm{~K} \mathrm{XbaI}$ and $50 \mathrm{~K}$ HindIII) was used to examine 116,204 SNPs according to the manufacturer's protocol 
(Affymetrix, Santa Clara, Calif., USA). GeneChip images were analyzed using GeneChip ${ }^{\circledR}$ DNA Analysis Software (version 3.0, Affymetrix). The quality control measures used to assess chip reliability are shown in online suppl. table 1 (for all online suppl. material, see www.karger.com/doi/10.1159/000326692). Briefly, samples with call rates below $92 \%$ were repeated. DNA provided by Affymetrix was included with each batch of samples as a positive control. Concordance of this positive control with known genotypes always exceeded $99 \%$, and the sex of each study sample was correctly identified by heterozygosity of the X chromosome. Additionally, 31 SNPs were replicated on both XbaI and HindIII arrays to ensure that samples were not switched; the median concordance between these 31 SNPs on the 2 arrays was $100 \%$ for each sample. SNPs that were monomorphic in this data set $(n=9,323)$ were removed, along with SNPs with call rates of less than $80 \%$ ( $\mathrm{n}=1,864$ ). An additional 2,363 SNPs located on the X chromosome were not included in this analysis. The remaining 102,654 polymorphisms were assessed to determine if the observed genotype frequencies were consistent with Hardy-Weinberg equilibrium using $\chi^{2}$ tests. Markers that deviated from Hardy-Weinberg equilibrium $(\mathrm{p}<0.05)$ in the control subjects were excluded. In total, 93,508 autosomal SNPs passed all quality control measures and proceeded to further analysis.

$\chi^{2}$ tests were used to assess allelic and genotypic associations between a marker and CFS in empirically defined CFS cases and controls. Potentially confounding factors, including age, sex, body mass index and depression history, were examined for association with CFS, but no adjustments were needed. All $p$ values were estimated using 10,000 Monte Carlo simulations under the null hypothesis of no association. To examine if population stratification was present in the sample, the genomic control method [26] was used.

\section{Gene Expression}

Gene expression was evaluated using the MWG 20K human array A (MWG Biotech, Ebersberg, Germany), which contains oligonucleotide features representing 20,160 genes. The laboratory procedure used in the expression study has been described previously [27]. Briefly, DNase I-treated total RNA $(1 \mu \mathrm{g})$ was reverse transcribed into biotinylated cDNA for hybridization to microarrays using the Ventana Discovery system and ChipMap ${ }^{\mathrm{TM}} \mathrm{kit}$ (Ventana Medical Systems, Ariz., USA). Hybridization signals were detected using antibiotin antibodies conjugated to resonance light-scattering RLS ${ }^{\mathrm{TM}}$ gold particles and a GSD-501 scanner (Genicon Sciences Corporation, Calif., USA). Signal intensity was quantified using ArrayVision ${ }^{\mathrm{TM}}$ RLS image analysis software (Genicon Sciences).

Microarray data analysis was conducted using ArrayTrack version 3.4.0 (http://www.fda.gov/nctr/science/centers/toxicoinformatics/ArrayTrack/). The intensity data were normalized using a global median scale to 1,000 and then $\log _{2}$ transformed. Genes differentially expressed between CFS and controls were assessed using a significance criteria of $\mathrm{p}<0.05$ (Welch test) and a fold change $>4$ with an average gene intensity filtering of 1,000 across all 62 hybridizations. Molecular function and associated pathways of the differentially expressed genes were extracted using Gene Ontology For Function Analysis (GOFFA), Kyoto Encyclopedia of Genes and Genomes (KEGG) and PathArt (proprietary pathway database from Jubilant Biosystems Ltd.) as implemented in ArrayTrack. Genes with scores yielding p values $<0.01$
Table 1. Demographic characteristics of the subjects in the study

\begin{tabular}{lcl}
\hline & $\begin{array}{l}\text { Controls } \\
(\mathrm{n}=40)\end{array}$ & $\begin{array}{l}\text { CFS subjects } \\
(\mathrm{n}=40)\end{array}$ \\
\hline Female, \% & 90.0 & 82.5 \\
Age, years (mean \pm SD) & $50.6 \pm 7.4$ & $50.7 \pm 8.7$ \\
BMI (mean \pm SD) & $29.3 \pm 5.3$ & $29.4 \pm 4.5$ \\
Current MDD, \% & 0.0 & 7.5 \\
Lifetime MDD, \% & 5.0 & 15.0 \\
Lifetime MDDM, \% & 15.0 & 22.5 \\
\hline
\end{tabular}

$\mathrm{BMI}=$ Body mass index $\mathrm{MDD}=$ major depressive disorder; $\mathrm{MDDM}=$ major depressive disorder with melancholia.

were considered biologically relevant by GOFFA. We also used Ingenuity Pathways Analysis (IPA; Ingenuity Systems Inc., Calif., USA) to identify molecular networks among the differentially expressed genes.

\section{Post hoc Analysis and Multiple Test Correction}

Fisher's combined probability test was used to incorporate the SNP association and gene expression results for each gene that reached significance independently in each experiment. A Bonferroni correction was then applied to account for the total number of possible tests.

\section{Results}

\section{SNP Association Study}

Subjects with CFS did not differ significantly from nonfatigued controls with respect to age, sex, body mass index or history of major depressive disorder or melancholic depression (table 1). A genomic control correction was used to evaluate potential population stratification. The mean (0.74) and median (0.29) $\chi^{2}$ values for over 50,000 unassociated SNPs were calculated and suggested no evidence that population stratification confounded the results. Allelic association tests revealed 3,234 SNPs associated with CFS with a p value $<0.05$ and 65 SNPs with a p value $<0.001$ (table 2). Regions of interest included chromosomes 1, 2, 6, 7, 10, 11, 12 and 13 and an extended area of association on chromosome 19. Thirty-five of these SNPs are located in or near 33 unique genes, and the remainder are located near expressed sequence tags $(\mathrm{n}=10)$ or in regions with no known genes $(\mathrm{n}=20$; table 2).

Several associated polymorphisms reside in or near known genes of particular relevance to CFS (table 2). For example, in GRIK2, the C allele of rs2247218 and the G 
Table 2. Allele frequencies and location of SNPs associated with CFS ( $\mathrm{p}<0.001)$

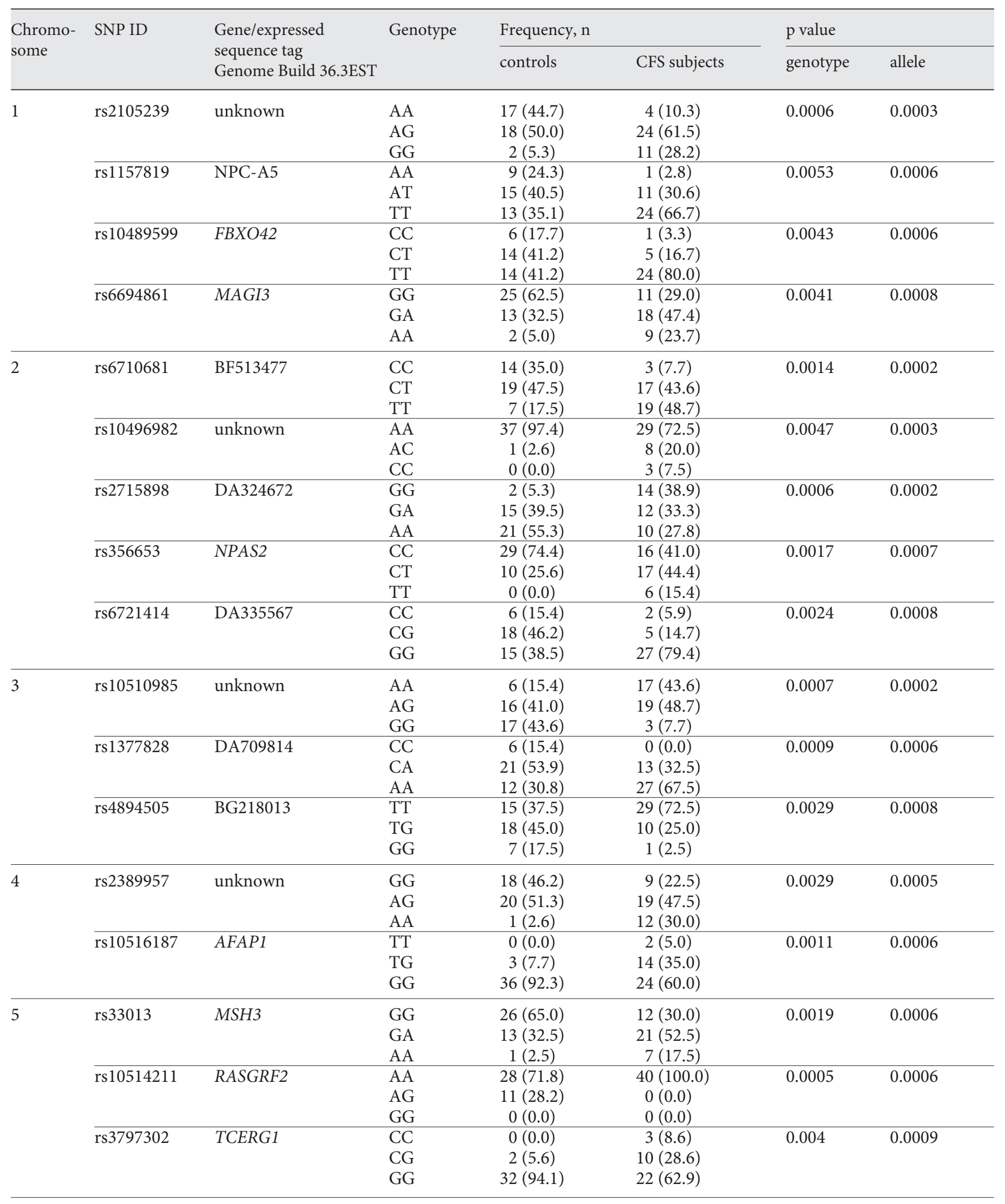


Table 2 (continued)

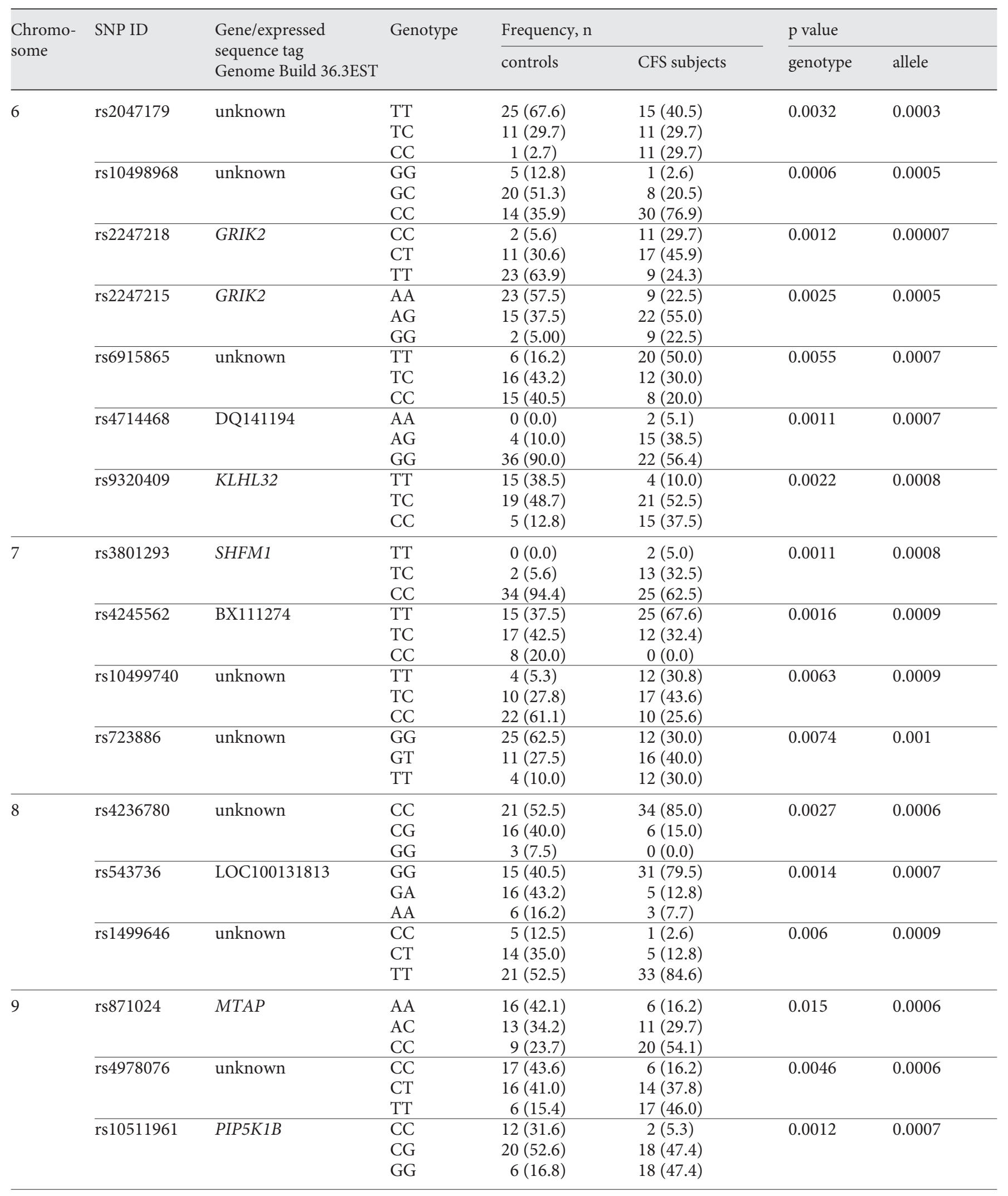


Table 2 (continued)

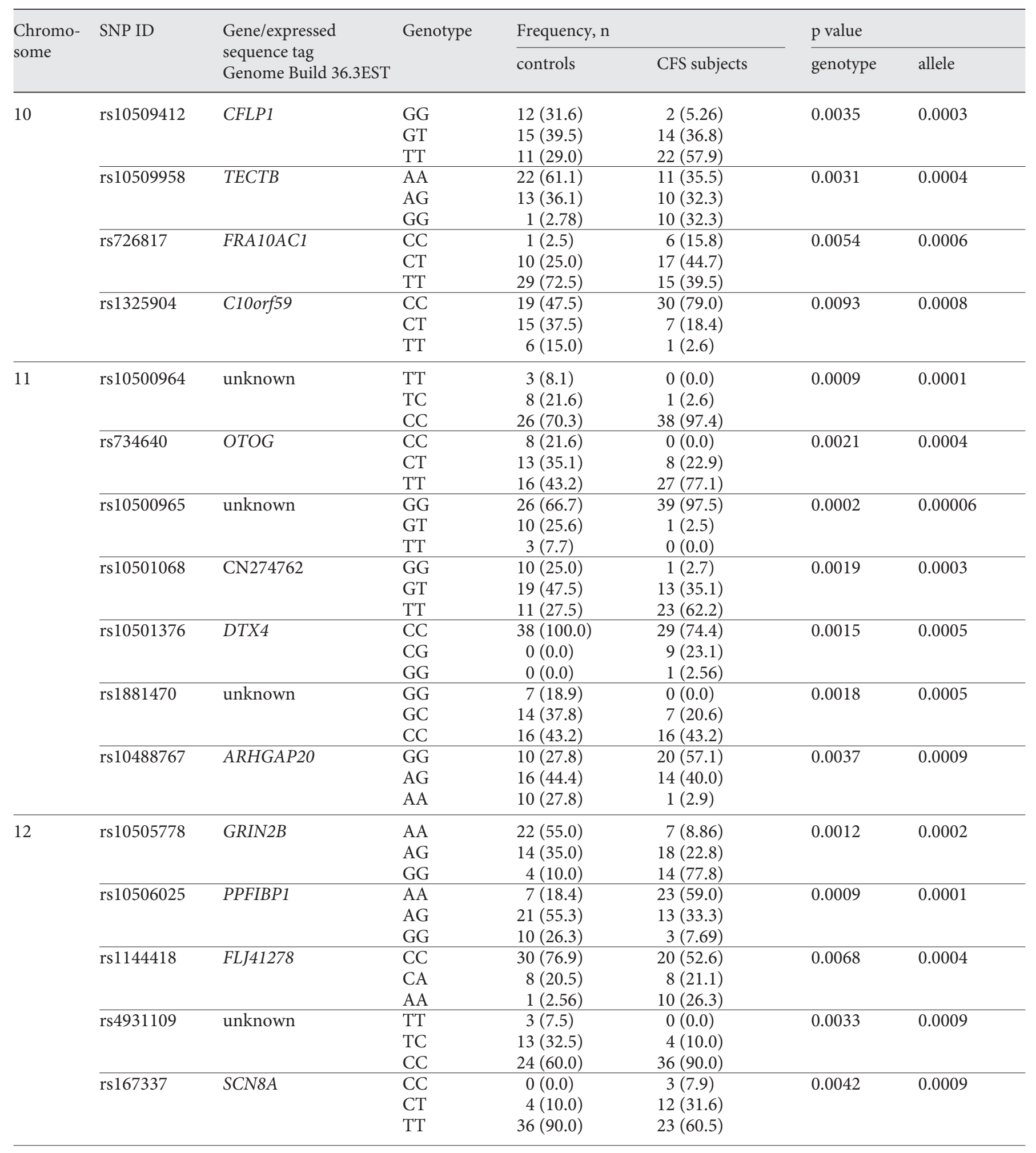


Table 2 (continued)

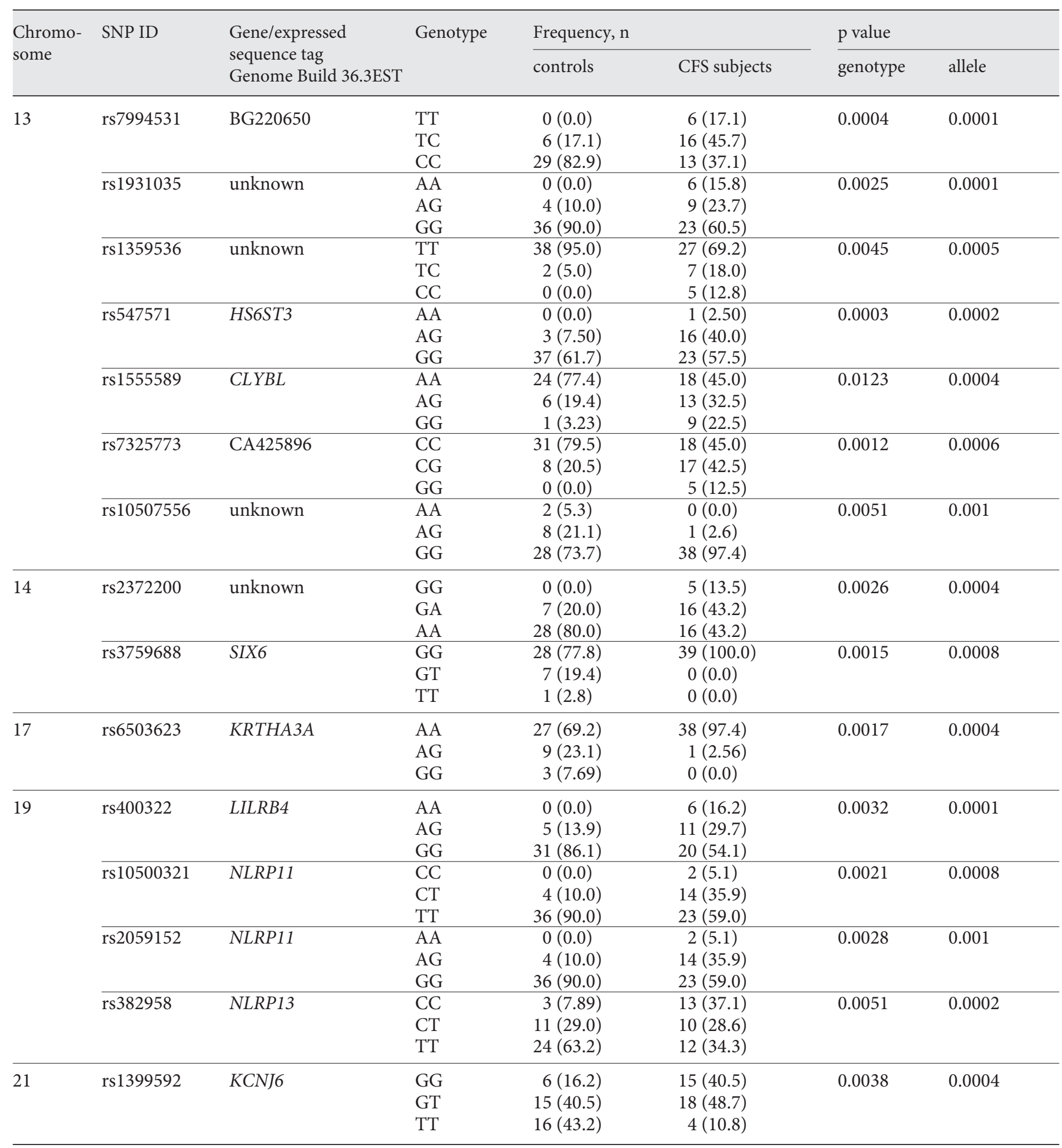

Mapping of SNPs to respective chromosomes, genes or expressed sequence tags was accomplished with NCBI Genome Build 36.3. Values in parentheses represent percentages. 
allele of rs 2247215 were more common in CFS cases (50.0\%) than in controls (24.0\%; $0.00007<\mathrm{p}<0.0005)$; both SNPs are in linkage disequilibrium. In glutamate receptor, ionotropic, N-methyl-D-aspartate 2B (GRIN2B), the $G$ allele of rs 10505778 was more common in CFS subjects $(59.0 \%)$ than in controls $(27.5 \%$; $p=0.0002)$. We also observed associated polymorphisms in 3 immune response genes, namely NLR family, pyrin domain-containing (NLRP) 11 and 13 and leukocyte immunoglobulin-like receptor, subfamily B, member 4 (LILRB4), which are located in a 1.28-megabase region of chromosome 19, which also contains several SNPs associated with CFS. The A allele of rs400322 (LILRB4) was more common in CFS subjects (31.1\%) than in controls $(6.9 \% ; \mathrm{p}=0.0001)$, and the C allele of rs382958 (NLRP13) was also more common in CFS subjects (51.4\%) than in controls $(22.4 \%$; $\mathrm{p}=0.0002)$. In NLRP11, the $\mathrm{C}$ allele of rs10500321 and the A allele of rs2059152 were also associated with CFS $(0.001<\mathrm{p}<0.0008)$. Finally, the $\mathrm{T}$ allele of rs356653, an SNP located in NPAS2, a gene implicated in circadian regulation, was more common in subjects with CFS ( $\mathrm{p}=$ 0.0007).

\section{Gene Expression Analysis}

The demographics of those subjects for whom we obtained gene expression data did not differ significantly from those included in the association study described above (data not shown). One hundred and sixty-five genes were differentially expressed between CFS subjects and controls (online suppl. table 2). Once again, genes of particular relevance to CFS were identified in this analysis. For example, hypocretin receptor 1 (HCRTR1) was one of the highly upregulated genes (nearly 20 -fold; $\mathrm{p}=0.004$ ) in CFS subjects in this study. Glutamate receptor, metabotropic 1 (GRM1) was upregulated 4.9-fold $(\mathrm{p}=0.046)$, and GRM4 was downregulated more than 10 -fold $(\mathrm{p}=0.0093)$. GRIK2 was also downregulated (10-fold; $\mathrm{p}=0.015)$. The mRNA expression of killer cell immunoglobulin-like receptor, 2 domains, long cytoplasmic tail 4 and 5 (KIR2DL4 and KIR2DL5) was 5 times lower for both in CFS subjects compared to controls $(\mathrm{p}=0.024$ and $\mathrm{p}=0.030$, respectively). Hypoxia-inducible factor 1 , alpha subunit (HIF1A) was downregulated 6-fold $(\mathrm{p}=0.026)$ in CFS subjects. Finally, NPAS2 was upregulated (nearly 10 -fold; $\mathrm{p}=0.027)$ in CFS subjects. However, only GRIK2 and NPAS2 were associated with CFS in both the SNP association and mRNA expression experiments.

Differentially expressed genes were then evaluated using GOFFA, KEGG, PathArt and IPA to explore gene function and molecular networks. Among these genes,
GOFFA yielded 42 genes $(\mathrm{p}<0.01)$ with a known molecular function, including GRIK2 and NPAS2 (online suppl. table 2). KEGG analysis of these 42 genes indentified 5 pathways $(\mathrm{p}<0.05)$, including neuroactive ligand-receptor interaction (HSA04080; APLNR, EDNRB, GRIK2, GRM1, GRM4, HCTRR1, OPRM1, TAAR9), taste transduction (HAS04742; TAS2R10, TAS2R9), leukocyte transendothelial migration (HSA04670; ACTN1, CTNNA1, CXCR4, PXN), adherens junctions (HSA04520; ACTN1, CTNNA1, SORBS1) and regulation of actin cytoskeleton (HSA04810; ACTN1, CFL1, SPTAN1). Similarly, PathArt analysis identified several pathways, notably chemotaxis (CXCR4, PXN), amyloid beta-peptide (NPAS2, PXN) and cell-cell (GRIK2, GRIM1, GRIM4, HCRTR1) signaling pathways. IPA organized the differentially expressed genes into 5 molecular networks (scores $\geq 20$ including a minimum of 10 focus molecules; online suppl. table 3 ).

Two genes, GRIK2 and NPAS2, reached significance independently in the SNP association and gene expression studies. We used Fisher's combined probability test to incorporate the SNP association and gene expression results for these two genes. Because the two polymorphisms in GRIK2 that were associated with CFS were in linkage disequilibrium and were therefore not independent, only the result from rs 2247218 was used. The results of this meta-analysis increased the magnitude of the associations for both GRIK2 ( $\mathrm{p}=0.000016)$ and NPAS2 $(\mathrm{p}=0.00022)$. However, 3,593 genes were common to both the SNP and expression analyses and are expressed in both the blood and brain according to the Body Atlas data implemented in MetaCore (GeneGo). Using a stringent Bonferroni correction for the number of possible genes tested, NPAS2 $(\mathrm{p}=0.55)$ did not retain significance while GRIK2 ( $\mathrm{p}=0.054)$ remained suggestive of an association.

\section{Discussion}

Convergent functional genomics allowed the identification of novel candidate genes, GRIK2 and NPAS2, involved in glutamatergic neurotransmission and the circadian rhythm, respectively, that are potentially associated with CFS. Polymorphisms in GRIK2 were associated with CFS, and expression of GRIK2 mRNA was decreased in CFS subjects. Also, a polymorphism in NPAS2 was more common in subjects with CFS, while NPAS2 expression was increased in CFS subjects. A recent computational study suggested that genes that are highly differentially 
expressed are more likely to harbor disease-associated DNA variants. By determining differential expression ratios (DERs) using all of the data sets $(>476)$ in the human Gene Expression Omnibus database, Chen et al. [28] demonstrated that genes with a DER $>0.55$ are 2.5 times more likely to harbor disease-associated genetic variants than constitutively expressed genes. We used the FitSNP database developed by Chen et al. [28] to determine DER values for GRIK2 and NPAS2. High DER values $(>0.55)$ for both GRIK2 (0.56) and NPAS2 (0.64) lend another level of analytical support to our conclusion that these genes warrant further examination as potential contributors to CFS pathophysiology. These associations must be confirmed in independent populations of subjects with CFS.

It is interesting to note that both GRIK2 and NPAS2 have been reported to be associated with impaired cognition as well as memory and sleep, 2 of the hallmark symptoms of CFS $[29,30]$. The association of these two genes with some of the hallmark symptoms of CFS, which overlaps with psychiatric illness, including mood and anxiety disorders [31], suggests that while these conditions can be clinically distinct, they may share neurobiological underpinnings. In support of this, we observed associations between CFS and genetic polymorphisms in 2 glutamate receptors, GRIK2 and GRIN2B. We also observed associations between CFS and mRNA expression of GRIK2, GRM1 and GRM4. Polymorphisms in glutamate receptors (GRIK2, GRIN2B) have been associated with a number of neuropsychiatric disorders such as autism [32], Huntington's disease [33], attention deficit/hyperactive disorder [34], obsessive compulsive disorder [35], antidepressant treatment-emergent suicidal ideation [36] and antipsychotic-induced weight gain [37] but have never been investigated in CFS. Among these polymorphisms, SNPs associated with suicidal ideation in patients being actively treated for depression (rs2518224) [36] reside in the same intron of GRIK2 that harbors the polymorphisms associated with CFS in this study (rs2247218 and rs2247215). Interestingly, the GRIN2B polymorphism (rs10505778) associated with CFS was also associated with antipsychotic-induced weight gain [37]. Since glutamate receptors mediate most excitatory neurotransmission in the brain and play an important role in learning, memory and mood regulation, the association of genetic variants in glutamate receptors as well as previous results implicating an association between chronic pain and mental fatigue and reduced glutamate uptake [38] support a shared role for the glutamatergic system in several cellular and cognitive processes involved in CFS, depression and autism.

Genomic Studies of CFS
Altered functioning of the endogenous circadian clock can lead to neurobiological, behavioral and psychiatric deteriorations. Multiple polymorphisms in genes involved in regulation of the circadian clock have been associated with sleep disorders and psychiatric diseases [39, 40]. In this study, we observed an association between CFS and a polymorphism (rs356653) in NPAS2, a member of the basic helix-loop-helix PAS family of transcription factors that is a central component of the molecular circadian oscillator. NPAS2 heterodimerizes with BMAL1 and functions as a positive element of the circadian system to drive the transcription of clock-controlled genes $[39,41]$. NPAS2 may function primarily as part of a molecular clock operating in the forebrain, but being a para$\log$ of $C L O C K$, it can also substitute for CLOCK in the master clock in the hypothalamic suprachiasmatic nuclei to regulate circadian rhythmicity [41, 42]. NPAS2 has been associated with cued and contextual memory [43] and may function as a transcriptional regulator of nonrapid eye movement sleep in mice $[44,45]$. Polymorphisms in NPAS2 have been associated with multiple psychiatric disorders. For example, variants have been associated with autism (rs1811399) [46], winter depression (rs11541353) [47], mood disorders and schizophrenia (rs13025524 and rs11123857) $[48,49]$ as well as breast cancer and non-Hodgkin's lymphoma (NHL) [50]. The associations between NPAS2 variants and CFS and NHL are interesting in the context of anecdotal reports suggesting that CFS may predispose subjects to NHL [51]. While none of these studies report an association with a common NPAS2 polymorphism, these associations suggest an overlapping role for NPAS2 in CFS and psychiatric disorders, potentially via common symptomatology such as sleep architecture or metabolic imbalances [52].

Associated polymorphisms in GRIK2 (rs2247218 and rs2247215) and NPAS2 (rs356653) are located in the first and second introns of their respective genes. Since these SNPs are in introns, their impact on gene function and ultimately the associated phenotype is not immediately clear. These markers may be in linkage disequilibrium with other causative markers or they may play a direct role in gene expression by affecting transcription factor binding, alternative splicing or microRNA production. Of the 2 GRIK2 SNPs, rs2247215 showed in silico evidence for alteration of consensus binding sites for 2 overlapping transcription factors, GATA1 and EVI1. The G allele of rs2247215 creates a consensus binding site for GATA1, suggesting that GRIK2, like other GATA1-repressed genes, may be repressed through the participation of polycomb repressive complex 2 [53]. This potential role of

Neuropsychobiology 2011;64:183-194 
rs 2247215 is consistent with the association of its $G$ allele with CFS and decreased expression of GRIK2 in CFS subjects. In silico analysis also identified a binding site for RFX1 transcription factor at rs356653 (NPAS2). The T allele of rs356653 is likely to disrupt RFX1 binding. RFX1 may be a transcriptional repressor of NPAS2 [54], and if so the $\mathrm{T}$ allele would lead to increased NPAS2 expression by disrupting RFX1 binding. This interpretation is consistent with our findings that the $\mathrm{T}$ allele is associated with CFS and increased expression of NPAS2 in CFS. However, experimental validation is required to support the role of rs2247215 and rs356653 variants in gene expression.

Associations between CFS and polymorphisms in LILRB4, NLRP11 and NLRP13 were observed, but we observed no difference in mRNA expression for these genes. However, only probes for LILRB 4 were available on the expression array that was used, so it remains unclear whether or not NLRP11 and NLRP13 are differentially expressed in subjects with CFS. Interestingly, KIR2DL4 and KIR2DL5, which are located between LILRB 4 and NLRP13, exhibited decreased expression in subjects with CFS. These two genes have been associated with Gulf War illness [55], whose symptoms resemble that of CFS in terms of fatigue, musculoskeletal discomfort and cognitive dysfunction. All of these genes are encoded within the leukocyte receptor complex on human chromosome 19q13. Studies suggest that postinfective fatigue persists in a subset of patients following infection by viral or nonviral microorganisms [56]. It is possible that individuals with a genetic susceptibility in the leukocyte receptor complex may develop CFS in response to a serious immune challenge.

Gene expression analysis identified two genes potentially replicating earlier discoveries of pathways or genes associated with CFS or postinfective fatigue syndrome in other populations. This study identified an orexin/hypocretin receptor, HCRTR1, as one of the highly upregulated genes (nearly 20 -fold) in CFS subjects. A functionally related gene, hypocretin receptor 2 (HCRTR2), was found to be positively correlated with sleep in subjects with postinfective fatigue in Australia [57]. HIF1A, which was downregulated 6-fold in CFS subjects in this study, was also reported to be downregulated in CFS subjects in the UK [7]. Based on these replicated gene expression array results and the reported interaction between orexin signaling and HIF1 activity [58], functional studies on the role of these orexin receptors and HIF1A in the pathogenesis of CFS are warranted.

While the present study has considerable strengths, including recruitment of subjects from the general population and rigorous clinical evaluations of both cases and controls, the study is limited by the small sample size and the fact that the statistical thresholds for both our genetic association and gene expression results are not stringent enough to achieve experiment-wide significance on their own. However, the statistical thresholds utilized in this study are comparable to or more stringent than those in recent studies that undertook a convergent functional genomics approach to identify viable candidate genes for further study from multiple lines of evidence [17-19]. Another limitation is the use of SNP and expression arrays that are not comprehensive of the genome, thus limiting coverage and making it less likely to identify polymorphisms that directly influence gene expression. However, it should be noted that the polymorphisms reported in this study achieved a greater level of statistical significance than those of any candidate gene study of CFS published to date. Further, GRIK2 and NPAS2 are differentially expressed, providing proof of principle support for the use of convergent functional genomics approaches for studies that would otherwise be underpowered, as well as support for further investigations of these genes for their potential contribution to the pathophysiology of CFS. Nonetheless, false-positive (type I error) results remain a possibility because of population stratification, multiple comparisons, the complexity and heterogeneity of CFS and difficulties with reproducible case ascertainment. Using a genomic control method, we found no evidence of population stratification. While increasing the sample size and determining Bonferroni-corrected $\mathrm{p}$ values can be helpful to address multiple comparisons, these approaches alone may not be enough to address the complexity and heterogeneity of an illness like CFS [20]. Validating these findings will require replication of SNP and gene expression analysis in a replication study in a larger independent population. Further, examining genetic associations at measurable narrow subphenotypic levels may minimize falsepositive findings across multiple populations.

The expression analysis in the present study was based on PBMCs, which may not be directly involved in CFS pathogenesis. However, given the bidirectional communication between the central nervous and peripheral systems [59], changes in PBMC expression are relevant as a reflection of that communication [22]. In addition, previous studies indicate that PBMCs express approximately $60 \%$ of the genes expressed in brain tissue $[12,60]$. The most compelling genes identified in this study are expressed in both the blood and brain tissues, but direct investigations of the influence of genetic variations on expression of these genes in multiple brain regions and blood cells remain to be performed. 
In conclusion, this study provides the first evidence for a role of genes involved with glutamatergic neurotransmission (GRIK2) and circadian rhythm (NPAS2) in CFS. Since GRIK2 and NPAS2 were identified from convergent results from complementary SNP and gene expression analyses, additional investigation in independent populations of CFS subjects is warranted. Replication of these findings will open novel avenues for the study of CFS pathogenesis. This study also underscores the utility of leveraging both SNP association and gene expression approaches to identify candidate genes from studies that otherwise would be underpowered.

\section{Acknowledgements}

Support for A.K. Smith was provided by the research participation program at the CDC, Division of High-Consequence Pathogens and Pathology, administered by the Oak Ridge Institute for Science and Education through an interagency agreement between the US Department of Energy and the CDC.

The authors wish to acknowledge the help of Supraja Narasimhan with genotyping and Dr. Virginia R. Falkenberg with in silico analyses of transcription factor binding, and the support and encouragement of Dr. William Reeves with this research project.

\section{References}

1 Klimas NG, Koneru AO: Chronic fatigue syndrome: inflammation, immune function, and neuroendocrine interactions. Curr Rheumatol Rep 2007;9:482-487.

$\checkmark 2$ Togo F, Natelson BH, Cherniack NS, FitzGibbons J, Garcon C, Rapoport DM: Sleep structure and sleepiness in chronic fatigue syndrome with or without coexisting fibromyalgia. Arthritis Res Ther 2008;10:R56.

$>3$ Van Den Eede F, Moorkens G, Van Houdenhove B, Cosyns P, Claes SJ: Hypothalamicpituitary-adrenal axis function in chronic fatigue syndrome. Neuropsychobiology 2007;55:112-120.

-4 Smith AK, Dimulescu I, Falkenberg VR, Narasimhan S, Heim C, Vernon SD, Rajeevan MS: Genetic evaluation of the serotonergic system in chronic fatigue syndrome. Psychoneuroendocrinology 2008;33:188-197.

$\checkmark 5$ Sullivan PF, Evengard B, Jacks A, Pedersen NL: Twin analyses of chronic fatigue in a Swedish national sample. Psychol Med 2005; 35:1327-1336.

-6 van de Putte EM, van Doornen LJ, Engelbert RH, Kuis W, Kimpen JL, Uiterwaal CS: Mirrored symptoms in mother and child with chronic fatigue syndrome. Pediatrics 2006; 117:2074-2079.

7 Kerr JR: Gene profiling of patients with chronic fatigue syndrome/myalgic encephalomyelitis. Curr Rheumatol Rep 2008;10: 482-491.

$>8$ Rajeevan MS, Smith AK, Dimulescu I, Unger ER, Vernon SD, Heim C, Reeves WC: Glucocorticoid receptor polymorphisms and haplotypes associated with chronic fatigue syndrome. Genes Brain Behav 2007;6:167-176.

-9 Saiki T, Kawai T, Morita K, Ohta M, Saito T, Rokutan K, Ban N: Identification of marker genes for differential diagnosis of chronic fatigue syndrome. Mol Med 2008;14:599-607.

$>10$ Sorensen B, Jones JF, Vernon SD, Rajeevan MS: Transcriptional control of complement activation in an exercise model of chronic fatigue syndrome. Mol Med 2008;15:34-42.
Harrison PJ, Weinberger DR: Schizophrenia genes, gene expression, and neuropathology: on the matter of their convergence. Mol Psychiatry 2005;10:40-68.

12 Middleton FA, Rosenow C, Vailaya A, Kuchinsky A, Pato MT, Pato CN: Integrating genetic, functional genomic, and bioinformatics data in a systems biology approach to complex diseases: application to schizophrenia. Methods Mol Biol 2007;401:337-364.

13 Vawter MP, Atz ME, Rollins BL, CooperCasey KM, Shao L, Byerley WF: Genome scans and gene expression microarrays converge to identify gene regulatory loci relevant in schizophrenia. Hum Genet 2006;119:558570.

14 Jiao H, Kaaman M, Dungner E, Kere J, Arner P, Dahlman I: Association analysis of positional obesity candidate genes based on integrated data from transcriptomics and linkage analysis. Int J Obes (Lond) 2008;32:816-825.

15 Glinsky GV: Integration of HapMap-based SNP pattern analysis and gene expression profiling reveals common SNP profiles for cancer therapy outcome predictor genes. Cell Cycle 2006;5:2613-2625.

16 Huang RS, Duan S, Bleibel WK, Kistner EO, Zhang W, Clark TA, Chen TX, Schweitzer AC, Blume JE, Cox NJ, Dolan ME: A genome-wide approach to identify genetic variants that contribute to etoposide-induced cytotoxicity. Proc Natl Acad Sci USA 2007;104:9758-9763.

17 Kurian SM, Le-Niculescu H, Patel SD, Bertram D, Davis J, Dike C Yehyawi N, Lysaker P, Dustin J, Caligiuri M, Lohr J, Lahiri DK, Nurnberger JI Jr, Faraone SV, Geyer MA, Tsuang MT, Schork NJ, Salomon DR, Niculescu AB: Identification of blood biomarkers for psychosis using convergent functional genomics. Mol Psychiatry 2011; 16:37-58.

18 Le-Niculescu H, Patel SD, Bhat M, Kuczenski R, Faraone SV, Tsuang MT, McMahon FJ, Schork NJ, Nurnberger JI Jr, Niculescu AB 3rd: Convergent functional genomics of genome-wide association data for bipolar disorder: comprehensive identification of candidate genes, pathways and mechanisms. Am J Med Genet B Neuropsychiatr Genet 2009;150B:155-181.

19 Le-Niculescu H, Kurian SM, Yehyawi N, Dike C, Patel SD, Edenberg HJ, Tsuang MT, Salomon DR, Nurnberger JI Jr, Niculescu AB: Identifying blood biomarkers for mood disorders using convergent functional genomics. Mol Psychiatry 2009;14:156-174.

20 Niculescu AB, Le-Niculescu H: The P-value illusion: how to improve (psychiatric) genetic studies. Am J Med Genet B Neuropsychiatr Genet 2010;153B:847-849.

21 Niculescu AB, Le-Niculescu H: Convergent functional genomics: what we have learned and can learn about genes, pathways, and mechanisms. Neuropsychopharmacology 2010;35:355-356.

22 Achiron A, Gurevich M: Peripheral blood gene expression signature mirrors central nervous system disease: the model of multiple sclerosis. Autoimmun Rev 2006;5:517-522.

-23 Reeves WC, Wagner D, Nisenbaum R, Jones JF, Gurbaxani B, Solomon L, Papanicolaou DA, Unger ER, Vernon SD, Heim C: Chronic fatigue syndrome - a clinically empirical approach to its definition and study. BMC Med 2005;3:19.

-24 Fukuda K, Straus SE, Hickie I, Sharpe MC, Dobbins JG, Komaroff A: The chronic fatigue syndrome: a comprehensive approach to its definition and study. International Chronic Fatigue Syndrome Study Group. Ann Intern Med 1994;121:953-959.

25 Reeves WC, Lloyd A, Vernon SD, Klimas N, Jason LA, Bleijenberg G, Evengard B, White PD, Nisenbaum R, Unger ER; International Chronic Fatigue Syndrome Study Group: Identification of ambiguities in the 1994 chronic fatigue syndrome research case definition and recommendations for resolution. BMC Health Serv Res 2003;3:25. 
-26 Devlin B, Roeder K: Genomic control for association studies. Biometrics 1999;55:9971004.

-27 Ojaniemi H, Evengard B, Lee DR, Unger ER, Vernon SD: Impact of RNA extraction from limited samples on microarray results. Biotechniques 2003;35:968-973.

-28 Chen R, Morgan AA, Dudley J, Deshpande T, Li L, Kodama K, Chiang AP, Butte AJ: FitSNPs: highly differentially expressed genes are more likely to have variants associated with disease. Genome Biol 2008;9:R170.

-29 Majer M, Welberg LA, Capuron L, Miller AH, Pagnoni G, Reeves WC: Neuropsychological performance in persons with chronic fatigue syndrome: results from a populationbased study. Psychosom Med 2008;70:829836.

- 30 Reeves WC, Heim C, Maloney EM, Youngblood LS, Unger ER, Decker MJ, Jones JF, Rye DB: Sleep characteristics of persons with chronic fatigue syndrome and non-fatigued controls: results from a population-based study. BMC Neurol 2006;6:41.

-31 Van Houdenhove B, Kempke S, Luyten P: Psychiatric aspects of chronic fatigue syndrome and fibromyalgia. Curr Psychiatry Rep 2010;12:208-214.

32 Kim SA, Kim JH, Park M, Cho IH, Yoo HJ: Family-based association study between GRIK2 polymorphisms and autism spectrum disorders in the Korean trios. Neurosci Res 2007;58:332-335.

- 33 Zeng W, Gillis T, Hakky M, Djousse L, Myers RH, MacDonald ME, Gusella JF: Genetic analysis of the GRIK2 modifier effect in Huntington's disease. BMC Neurosci 2006;7: 62.

-34 Dorval KM, Wigg KG, Crosbie J, Tannock R, Kennedy JL, Ickowicz A, Pathare T, Malone M, Schachar R, Barr CL: Association of the glutamate receptor subunit gene GRIN2B with attention-deficit/hyperactivity disorder. Genes Brain Behav 2007;6:444-452.

35 Arnold PD, Rosenberg DR, Mundo E, Tharmalingam S, Kennedy JL, Richter MA: Association of a glutamate (NMDA) subunit receptor gene (GRIN2B) with obsessivecompulsive disorder: a preliminary study. Psychopharmacology (Berl) 2004;174:530538.

-36 Laje G, Paddock S, Manji H, Rush AJ, Wilson AF, Charney D, McMahon FJ: Genetic markers of suicidal ideation emerging during citalopram treatment of major depression. Am J Psychiatry 2007;164:1530-1538.

- 37 Need AC, Keefe RS, Ge D, Grossman I, Dickson S, McEvoy JP, Goldstein DB: Pharmacogenetics of antipsychotic response in the CATIE trial: a candidate gene analysis. Eur J Hum Genet 2009;17:946-957.

38 Ronnback L, Hansson E: On the potential role of glutamate transport in mental fatigue. J Neuroinflammation 2004;1:22.
39 Barnard AR, Nolan PM: When clocks go bad: neurobehavioural consequences of disrupted circadian timing. PLoS Genet 2008; 4:e1000040.

40 Wulff K, Porcheret K, Cussans E, Foster RG: Sleep and circadian rhythm disturbances: multiple genes and multiple phenotypes. Curr Opin Genet Dev 2009;19:237-246.

41 DeBruyne JP, Weaver DR, Reppert SM: CLOCK and NPAS2 have overlapping roles in the suprachiasmatic circadian clock. Nat Neurosci 2007; 10:543-545.

42 Reick M, Garcia JA, Dudley C, McKnight SL: NPAS2: an analog of clock operative in the mammalian forebrain. Science 2001;293: 506-509.

43 Garcia JA, Zhang D, Estill SJ, Michnoff C, Rutter J, Reick M, Scott K, Diaz-Arrastia R, McKnight SL: Impaired cued and contextual memory in NPAS2-deficient mice. Science 2000;288:2226-2230.

-44 Franken P, Dudley CA, Estill SJ, Barakat M, Thomason R, O'Hara BF, McKnight SL: NPAS2 as a transcriptional regulator of nonrapid eye movement sleep: genotype and sex interactions. Proc Natl Acad Sci USA 2006; 103:7118-7123.

45 Wisor JP, Pasumarthi RK, Gerashchenko D, Thompson CL, Pathak S, Sancar A, Franken P, Lein ES, Kilduff TS: Sleep deprivation effects on circadian clock gene expression in the cerebral cortex parallel electroencephalographic differences among mouse strains. J Neurosci 2008;28:7193-7201.

46 Nicholas B, Rudrasingham V, Nash S, Kirov G, Owen MJ, Wimpory DC: Association of Per1 and Npas2 with autistic disorder: support for the clock genes/social timing hypothesis. Mol Psychiatry 2007;12:581-592.

47 Partonen T, Treutlein J, Alpman A, Frank J, Johansson C, Depner M, Aron L, Rietschel M, Wellek S, Soronen P, Paunio T, Koch A, Chen P, Lathrop M, Adolfsson R, Persson ML, Kasper S, Schalling M, Peltonen L, Schumann G: Three circadian clock genes Per2, Arntl, and Npas2 contribute to winter depression. Ann Med 2007;39:229-238.

48 Mansour HA, Talkowski ME, Wood J, Chowdari KV, McClain L, Prasad K, Montrose D, Fagiolini A, Friedman ES, Allen MH, Bowden CL, Calabrese J, El-Mallakh RS, Escamilla M, Faraone SV, Fossey MD, Gyulai L, Loftis JM, Hauser P, Ketter TA, Marangell LB, Miklowitz DJ, Nierenberg AA, Patel J, Sachs GS, Sklar P, Smoller JW, Laird N, Keshavan M, Thase ME, Axelson D, Birmaher B, Lewis D, Monk T, Frank E, Kupfer DJ, Devlin B, Nimgaonkar VL: Association study of 21 circadian genes with bipolar I disorder, schizoaffective disorder, and schizophrenia. Bipolar Disord 2009;11:701-710.
49 Soria V, Martínez-Amorós E, Escaramís G, Valero J, Pérez-Egea R, García C, GutiérrezZotes A, Puigdemont D, Bayés M, Crespo JM, Martorell L, Vilella E, Labad A, Vallejo J, Pérez V, Menchón JM, Estivill X, Gratacòs M, Urretavizcaya M: Differential association of circadian genes with mood disorders: CRY1 and NPAS2 are associated with unipolar major depression and CLOCK and VIP with bipolar disorder. Neuropsychopharmacology 2010;35:1279-1289.

50 Hoffman AE, Zheng T, Ba Y, Zhu Y: The circadian gene NPAS2, a putative tumor suppressor, is involved in DNA damage response. Mol Cancer Res 2008;6:1461-1468.

-51 Levine PH, Peterson D, McNamee FL, O’Brien K, Gridley G, Hagerty M, Brady J, Fears T, Atherton M, Hoover R: Does chronic fatigue syndrome predispose to nonHodgkin's lymphoma? Cancer Res 1992; 52(19 suppl):5516s-5518s, discussion 5518s$5521 \mathrm{~s}$

52 Bass J, Takahashi JS: Circadian integration of metabolism and energetics. Science 2010; 330:1349-1354.

53 Yu M, Riva L, Xie H, Schindler Y, Moran TB, Cheng Y, Yu D, Hardison R, Weiss MJ, Orkin $\mathrm{SH}$, Bernstein BE, Fraenkel E, Cantor AB: Insights into GATA-1-mediated gene activation versus repression via genome-wide chromatin occupancy analysis. Mol Cell 2009;36:682-695.

54 Zhao M, Sun Y, Gao F, Wu X, Tang J, Yin H, Luo Y, Richardson B, Lu Q: Epigenetics and SLE: RFX1 downregulation causes CD11a and CD70 overexpression by altering epigenetic modifications in lupus CD4+ T cells. J Autoimmun 2010;35:58-69.

55 Whistler T, Fletcher MA, Lonergan W, Zeng XR, Lin JM, Laperriere A, Vernon SD, Klimas NG: Impaired immune function in Gulf War Illness. BMC Med Genomics 2009;2:12.

56 Hickie I, Davenport T, Wakefield D, VollmerConna U, Cameron B, Vernon SD, Reeves WC, Lloyd A; Dubbo Infection Outcomes Study Group: Post-infective and chronic fatigue syndromes precipitated by viral and non-viral pathogens: prospective cohort study. BMJ 2006;333:575.

57 Vernon SD, Nicholson A, Rajeevan M, Dimulescu I, Cameron B, Whistler T, Lloyd A: Correlation of psycho-neuroendocrineimmune (PNI) gene expression with symptoms of acute infectious mononucleosis. Brain Res 2006;1068:1-6.

58 Sikder D, Kodadek T: The neurohormone orexin stimulates hypoxia-inducible factor-1 activity. Genes Dev 2007;21:2995-3005.

59 Silverman MN, Pearce BD, Biron CA, Miller AH: Immune modulation of the hypothalamic-pituitary-adrenal (HPA) axis during viral infection. Viral Immunol 2005; 18:41-78.

-60 Nicholson AC, Unger ER, Mangalathu R, Ojaniemi H, Vernon SD: Exploration of neuroendocrine and immune gene expression in peripheral blood mononuclear cells. Brain Res Mol Brain Res 2004;129:193-197. 